\title{
Effect of carbon dioxide pneumoperitoneum on human renal cell carcinoma proliferation and metastasis in an orthotropic xenograft nude mouse model
}

\author{
Yuan-Zhuo Chen, Yun-Fei Xu, Jun-Hua Zheng
}

Department of Urology, Shanghai Tenth People's Hospital, Tongji University School of Medicine, Shanghai, China

Submitted: 8 March 2013

Accepted: 23 August 2013

Arch Med Sci 2014; 10, 5: 1041-1046

DOI: 10.5114 /aoms.2014.46222

Copyright $\odot 2014$ Termedia \& Banach

\section{Abstract}

Introduction: This study aimed to explore the effect of carbon dioxide $\left(\mathrm{CO}_{2}\right)$ pneumoperitoneum on tumor proliferation and metastasis in an orthotropic xenograft nude mice model of human renal cell carcinoma (RCC) and evaluate the safety of $\mathrm{CO}_{2}$ pneumoperitoneum laparoscopy for treating RCC.

Material and methods: RCC 786-0 cells were injected to establish an orthotropic xenograft model. Fifty nude mice were given orthotropic inoculations and randomized to five groups: group $\mathrm{A}$ (control); group $\mathrm{B}\left(\mathrm{CO}_{2}\right.$ pneumoperitoneum for $2 \mathrm{~h})$; group $\mathrm{C}\left(\mathrm{CO}_{2}\right.$ pneumoperitoneum for $\left.4 \mathrm{~h}\right)$; group $\mathrm{D}\left(\mathrm{CO}_{2}\right.$ pneumoperitoneum for $4 \mathrm{~h}$ and $24 \mathrm{~h}$ after waking); group $\mathrm{E}\left(\mathrm{CO}_{2}\right.$ pneumoperitoneum for $4 \mathrm{~h}$ and $48 \mathrm{~h}$ after waking). The proliferation status was observed in RCC specimens by immunohistochemical staining for Ki67. The protein levels of hypoxia-inducible factor- $1 \alpha$ (HIF-1 $\alpha$ ) and vascular endothelial growth factor (VEGF) were examined by western blotting.

Results: All groups showed similar Ki67-positive staining in RCC samples $(p>0.05)$. The relative expression of HIF- $1 \alpha$ and VEGF gradually increased in both group $B$ and group $C$, as compared with group $A$, but only the difference between group $C$ and group A reached statistical significance $(p<0.05)$. The protein levels of HIF- $1 \alpha$ and VEGF decreased in both group D and group E, as compared with group $B$ and group $C$; however, the differences between group $\mathrm{D}$, group $\mathrm{E}$, and group $\mathrm{A}$ did not reach statistical significance $(p>0.05)$. Conclusions: In an orthotropic xenograft nude mice model of RCC, $\mathrm{CO}_{2}$ pneumoperitoneum has no effect on expression of the cellular proliferation marker Ki67. However, $\mathrm{CO}_{2}$ pneumoperitoneum rapidly induces transient expression of HIF- $1 \alpha$ and VEGF. Thus, $\mathrm{CO}_{2}$ pneumoperitoneum laparoscopy may be a safe method for treating RCC.

Key words: carbon dioxide pneumoperitoneum, renal cell carcinoma, tumor proliferation, hypoxia-inducible factor- $1 \alpha$, vascular endothelial growth factor.

\section{Introduction}

While several studies have investigated the effects of simulated $\mathrm{CO}_{2}$ pneumoperitoneum on tumor proliferation- and metastasis-related factors in vitro and in vivo [1-11], none have explored the effects in an orthotropic xenograft nude mice model of renal cell carcinoma (RCC). In RCC, many molecules play key roles in tumor proliferation and metastasis via inducing endothelial cell hyperplasia and degradation of extracellular matrix, such as hypoxia-inducible factor- $1 \alpha$ (HIF-1 $\alpha$ ), cyclooxygenase

\author{
Corresponding author: \\ Jun-Hua Zheng \\ Department of Urology \\ Shanghai Tenth People's \\ Hospital \\ Tongji University \\ School of Medicine \\ Yanchang Road 301 \\ 200072 Shanghai, China \\ Phone: +8618917684098 \\ E-mail: zhengjunhua1127@ \\ gmail.com
}


(COX), heparanase (HPA), vascular endothelial growth factor (VEGF), matrix metalloproteinases (MMPs) [12], vascular cell adhesion molecule 1 (VCAM-1) and E-cadherin. The abdominal cavity can be an oxygen-rich environment under normal physiological conditions. Since pneumoperitoneum with $100 \% \mathrm{CO}_{2}$ has been used during laparoscopy, it is necessary to study the effect of the induced hypoxic environment on local tissue during the surgical procedure. Ridgway et al. reported that the initial hypoxia during laparoscopic surgery was the most likely mechanism mediating a tumor's invasive tendencies [13]. Under anoxic conditions, HIF-1 is a critical regulator of various transcriptional responses, directly binding to multiple target genes in order to mediate adaptive responses to hypoxia. In malignant tumors, HIF-1 is known to play a key role in maintaining energy metabolism, promoting angiogenesis, and inducing proliferation and metastases [14-16]. A main subunit of HIF-1, HIF- $1 \alpha$, had been defined as a central mediator of RCC tumorigenesis [17]. In hypoxic tissues, HIF- $1 \alpha$ drives transcription of VEGF, which is one of the most important growth factors responsible for neovascularization. The process of tumor angiogenesis also plays key roles in tumor proliferation, invasion, and metastasis [18]. The Ki67 protein is a well-established cellular marker of proliferation, and the reflected tumor proliferative rate is related to tumor initiation, progression, metastases, and prognosis [19].

Thus, we examined HIF-1 $\alpha$, VEGF and Ki67 expression to explore the mechanism of $\mathrm{CO}_{2}$ influence on tumor proliferation and metastasis. In addition, the study was conducted to evaluate the safety of $\mathrm{CO}_{2}$ pneumoperitoneum laparoscopy for treating $\mathrm{RCC}$.

\section{Material and methods}

The RCC cell line 786-0 was obtained from the Shanghai Institutes for Biological Sciences, Institute of Biochemistry and Cell Biology at the Chinese Academy of Science Institute. Seventy $\mathrm{BALB} / \mathrm{c}$ nude mice were purchased from Shanghai SLAC Laboratory Animal Co., Ltd. The surgery department of our hospital provided the surgical $\mathrm{CO}_{2}$ insufflator (Olympus). The Ki67 cellular proliferation detection kit for immunohistochemical (IHC) staining was obtained from Nanjing KeyGen Biotech Co., Ltd. Primary antibodies against human HIF- $1 \alpha$ and VEGF were purchased from Santa Cruz Biotechnology. The experimental procedures and the animal use and care protocols were approved by the Committee on Ethical Use of Animals of Shanghai Tenth People's Hospital.

The $786-0$ cells $\left(1 \times 10^{7} / \mathrm{ml}\right)$ were suspended in RPMI 1640 medium without serum. The nude mice were anesthetized with a single intraperito- neal (i.p.) injection of $1 \%$ pentobarbital sodium (50 $\mu \mathrm{l} / 10 \mathrm{~g})$. A small vertical incision was made through the dorsal muscles of the body wall in the region of the left kidney. Cells $(0.1 \mathrm{ml} / \mathrm{mice})$ were injected into the renal capsule, and the muscles and skin were closed with silk sutures. Ten weeks later, tumors were removed for analysis. Three mice had died during the inoculation and two mice died during the tumor growth period. Finally, 50 tumor-implanted mice were individually tagged and randomized into control and test groups designated as group A, B, C, D and E consisting of 10 mice each.

In group $A$, the mice were anesthetized with a single i.p. injection of $1 \%$ pentobarbital sodium $(50 \mu \mathrm{l} / 10 \mathrm{~g})$ for $4 \mathrm{~h}$ and sacrificed by cervical dislocation. Each tumor was resected and divided into two parts, one of which was fixed with $4 \%$ paraformaldehyde and the other one was snap-frozen in liquid nitrogen. In groups B, C, D and E, the mice were anesthetized and a size 7 needle for the scalp vein was used as the Veress needle and inserted from the left side of the back to create the pneumoperitoneum. The needle was connected to the surgical $\mathrm{CO}_{2}$ insufflator by a plastic tube. Carbon dioxide pneumoperitoneum pressure was set to $15 \mathrm{~mm} \mathrm{Hg}, \mathrm{CO}_{2}$ flow to $60 \mathrm{ml} / \mathrm{min}$ and temperature to $37^{\circ} \mathrm{C}$ through the insufflator, after which $100 \% \mathrm{CO}_{2}$ insufflations were applied to the groups for 2 h, 4 h, 4 h, or 4 h, respectively. During this procedure, anesthesia was maintained by i.p. injection of $1 \%$ pentobarbital sodium. Mice from groups $D$ and $E$ were allowed to recover under normal conditions for $24 \mathrm{~h}$ and $48 \mathrm{~h}$ after waking, respectively, and then sacrificed by cervical spine dislocation. All tumor samples from groups $D$ and E were divided into two parts for fixing with $4 \%$ paraformaldehyde and storing in liquid nitrogen, respectively. During this process, one mouse in group $\mathrm{C}$ died during the $\mathrm{CO}_{2}$ insufflation and one mouse in group $D$ died of unknown causes during the recovery period.

Sections of the $4 \%$ formaldehyde-fixed paraffin-embedded sections were prepared and stained with hematoxylin and eosin $(\mathrm{H}+\mathrm{E})$ to evaluate the RCC and cell pleomorphism. One section was subjected to IHC staining of Ki67. After visualization by DAB substrate, microscopic images were captured and analyzed by the Leica Automatic Microscope and Image Analysis System. All data were calculated as mean and standard deviation (SD). Larger optical density (OD) values indicate stronger positivity, which reflects the proliferation state of a cell.

Relative expression of HIF- $1 \alpha$ and VEGF in tumor specimens was determined by western blotting. After estimating protein concentration by Coomassie Blue G250, $30 \mu \mathrm{g}$ of total protein was loaded and run on a $10 \%$ SDS-PAGE gel at $80 \mathrm{~V}$ for 
$30 \mathrm{~min}$, after which the voltage was increased to $100 \mathrm{~V}$ and the gel run until the bromophenol blue tracking dye reached the bottom. After transferring to nitrocellulose membranes at $120 \mathrm{~V}$ for $90 \mathrm{~min}$, the membranes were blocked with $2 \%$ bovine serum albumin overnight and washed with Tris-buffered saline (TBS; $10 \mathrm{mmol} / \mathrm{l}, \mathrm{pH}$ 7.4). The appropriate primary and secondary antibodies were then added and the membranes incubated for $2 \mathrm{~h}$. After washing with TBS, the immunoreactive bands were detected with chemiluminescence reagents, scanned by the Bio-Rad Gel Imaging System, and quantified by the accompanying Quantity One software. The relative expression level was calculated as the gray value of target bands divided by the gray value of the GAPDH band as an internal control.

\section{Statistical analysis}

All data were analyzed by SPSS 13.0 software. Significance of intergroup differences for the OD values of IHC staining and the relative protein expression was assessed by the ANOVA test. Values of $p<0.05$ were considered significant.

\section{Results}

\section{Tumor-implanted mice}

Obvious round- or oval-shaped tumors had formed around the left kidney by 10 weeks after inoculation. Tumors ranged in size from $0.5 \mathrm{~cm}$ to $2 \mathrm{~cm}$ in diameter $(1.3 \pm 0.4 \mathrm{~cm})$. Finally, 52 of 70 mice with a tumor burden survived the inoculation procedure and tumor growth period without developing dyscrasia. The overall tumor formation rate was $74.3 \%$. Renal parenchyma and capsule adhesion with tumor tissues and intra-abdominal metastasis were observed in 11 of the inoculated mice.

\section{$\mathrm{H}+$ E-stained sections and Ki67-positive expression}

$\mathrm{H}+\mathrm{E}$ staining showed obvious cell pleomorphism in tumor tissues and typical morphological features of RCC. Tumor samples from all groups showed positive staining for Ki67 expression (Figure 1). The OD values are listed in Table I. There was no significant difference among the mean OD values for any of the groups $(p>0.05)$.

\section{Western blotting analysis of HIF- $1 \alpha$ and VEGF expression}

The quantification of the relative expression of HIF- $1 \alpha$ and VEGF is presented in Figure 2. The expression of both HIF- $1 \alpha$ and VEGF was gradually increased in groups $B$ and $C$, as compared with group $A$. While the difference between group $A$ and group $B$ did not reach statistical significance $(p>0.05)$, the difference between group $C$ and group A did $(p<0.05)$. Compared with groups $B$ and $C$, groups $D$ and $E$ showed lower levels of HIF- $1 \alpha$ and VEGF expression. However, the levels in groups $D$ and $E$ were not significantly different from those in group $A(p>0.05)$.

\section{Discussion}

The safety of laparoscopy for treating malignancies remains controversial, especially in relation to whether the laparoscopic procedure itself
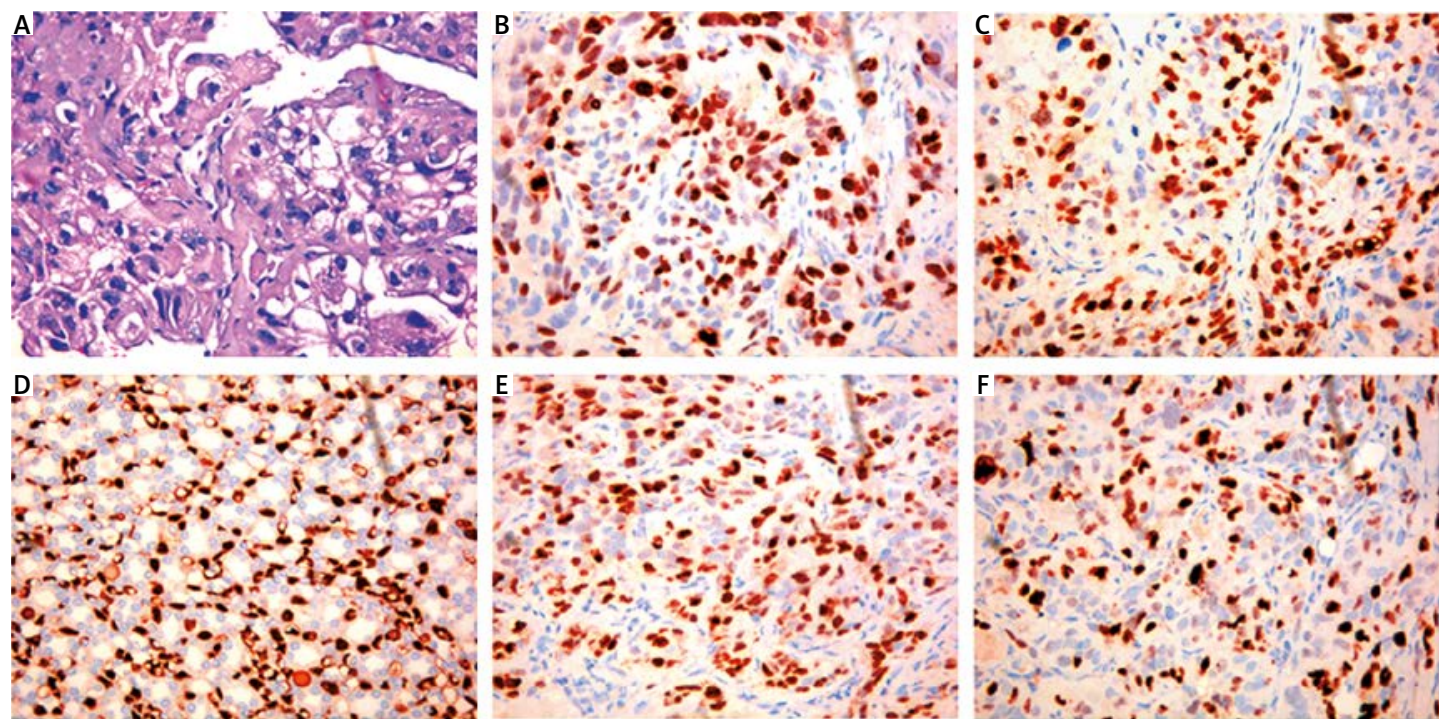

Figure 1. Representative $\mathrm{H}+\mathrm{E}$-stained tumor section and Ki67 expression in RCC tissues of control and $\mathrm{CO}_{2}$ pneumoperitoneum treated groups. A - Representative $\mathrm{H}+$ E-stained tumor section. B - Control group. $\mathbf{C}-\mathrm{CO}_{2}$ pneumoperitoneum for $2 \mathrm{~h}$. D - $\mathrm{CO}_{2}$ pneumoperitoneum for $4 \mathrm{~h}$. E- $\mathrm{CO}_{2}$ pneumoperitoneum for $4 \mathrm{~h}$ and $24 \mathrm{~h}$ after waking. $\mathrm{F}-\mathrm{CO}_{2}$ pneumoperitoneum for $4 \mathrm{~h}$ and $48 \mathrm{~h}$ after waking. All images are of magnification 200x 
Table I. OD values of Ki67 immunohistochemical staining

\begin{tabular}{|c|c|c|c|c|c|}
\hline \multirow[t]{2}{*}{ Characteristic } & \multicolumn{5}{|c|}{ OD values } \\
\hline & Group A $(n=10)$ & Group B $(n=10)$ & Group C $(n=9)^{*}$ & Group D $(n=9)^{*}$ & Group E $(n=10)$ \\
\hline & 1.2224 & 1.2151 & 1.2079 & 1.2732 & 1.2761 \\
\hline & 1.3190 & 1.2605 & 1.2351 & 1.2705 & 1.1864 \\
\hline & 1.1889 & 1.2715 & 1.1906 & 1.2606 & 1.2673 \\
\hline & 1.2605 & 1.2602 & 1.1433 & 1.3015 & 1.2961 \\
\hline & 1.2231 & 1.2723 & 1.2977 & 1.2051 & 1.2316 \\
\hline & 1.2452 & 1.1817 & 1.2854 & 1.2320 & 1.1943 \\
\hline & 1.2427 & 1.2674 & 1.1823 & 1.2302 & 1.2322 \\
\hline & 1.1932 & 1.2841 & 1.2696 & 1.1557 & 1.2015 \\
\hline & 1.2086 & 1.2006 & 1.2205 & 1.2461 & 1.1315 \\
\hline & 1.2524 & 1.1618 & & & 1.2964 \\
\hline Mean \pm SD & $1.2356 \pm 0.0381$ & $1.2375 \pm 0.0435$ & $1.2258 \pm 0.0512$ & $1.2416 \pm 0.0429$ & $1.2313 \pm 0.0537$ \\
\hline Value of $p^{\star *}$ & - & 0.93 & 0.65 & 0.78 & 0.84 \\
\hline
\end{tabular}

*One mouse in group $C$ died during the $\mathrm{CO}_{2}$ insufflation and one mouse in group $D$ died of unknown causes during the recovery period. ${ }^{\star *}$ Comparison between group $A$ and groups $B, C, D, E$, between all groups, $p=0.959$

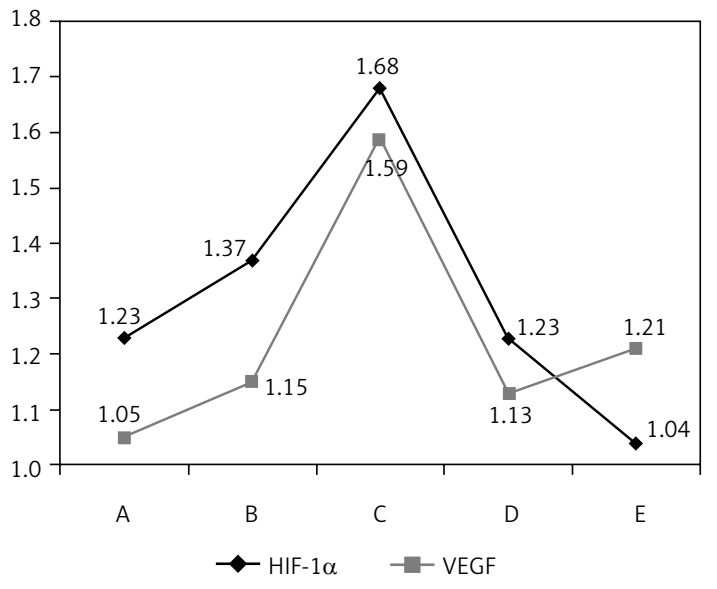

Figure 2. Relative protein expression of HIF-1 $\alpha$ (black) and VEGF (grey) in all groups

$C p<0.05$ and $B, D, E p>0.05$ vs. group $A$

can stimulate growth and/or induce metastasis of tumor cells. It has been reported that the laparoscopic $\mathrm{CO}_{2}$ pneumoperitoneum procedure can increase both abdominal pressure and hypoxia, as well as decrease the $\mathrm{pH}$ in the abdominal cavity [20]. Recent research has explored the potential effects of $\mathrm{CO}_{2}$ pneumoperitoneum on tumor metastasis and metastases during laparoscopy. The recent studies on $\mathrm{CO}_{2}$ pneumoperitoneum can mainly be divided into two groups: the first has investigated the effects of different $\mathrm{CO}_{2}$ pneumoperitoneum parameters on cancer cells' behavior using in vitro systems, while the second has evaluated the impact of various $\mathrm{CO}_{2}$ pneumoperi- toneum parameters in vivo using i.p. injection or xenograft tumor models. However, the ability of $\mathrm{CO}_{2}$ insufflations during laparoscopy to induce or inhibit tumor growth and metastases remains controversial [1-11].

The i.p. xenograft models, and not the orthotropic transplant tumor models, used in most of the animal studies were significantly different from the clinical tumor growth environments. In addition, very few studies have focused on the effect of $\mathrm{CO}_{2}$ pneumoperitoneum on RCC. Therefore, we designed the current study using an orthotropic xenograft nude mice model, which more closely mimics the clinical tumor environment. Our study also aimed to recapitulate the clinical $\mathrm{CO}_{2}$ pneumoperitoneum environment for treating RCC to the utmost extent. First, we simulated the RCC growth environment using a human RCC orthotropic xenograft. Second, two time points ( $2 \mathrm{~h}$ and $4 \mathrm{~h}$ ) were examined, because most laparoscopic procedures should be completed within $4 \mathrm{~h}$; for example, laparoscopic radical or partial nephrectomies for RCC should be finished within $2 \mathrm{~h}$. Third, $\mathrm{CO}_{2}$ insufflations at $15 \mathrm{~mm} \mathrm{Hg}$ pressure were used, since this is consistent with the common pressure used in the clinic.

At the cellular and tissue levels, there were no significant differences in Ki67 expression of the control and experimental groups of our study. At the protein level, $2 \mathrm{~h}$ of $\mathrm{CO}_{2}$ pneumoperitoneum increased HIF-1 $\alpha$ and VEGF (vs. control group, $p>0.05)$, in agreement with previous results. Cai et al. [21] reported that VEGF expression was significantly increased after $1 \mathrm{~h}$ of $\mathrm{CO}_{2}$ pneumo- 
peritoneum. In our RCC orthotropic xenograft model, with tumors located in the kidney where sufficient blood supply was available, the overall impact of pneumoperitoneum pressure on the tumor cell environment was expected to be less than that of an in vitro or i.p. injection model. The group administered $4 \mathrm{~h}$ of $\mathrm{CO}_{2}$ pneumoperitoneum showed significantly increased HIF-1 $\alpha$ and VEGF (vs. control group, $p<0.01$ ). However, the induced HIF- $1 \alpha$ and VEGF expression gradually decreased during the $24 \mathrm{~h}$ and $48 \mathrm{~h}$ recovery (vs. control group, $p>0.05$ ). Our results might reflect the normal physiological mechanisms of nude mice, in which $\mathrm{CO}_{2}$ pneumoperitoneum has no effect on tumor tissues with lower expression of HIF- $1 \alpha$ and VEGF. Thus, the effect of $\mathrm{CO}_{2}$ pneumoperitoneum on the changes of tumor proliferation and metastasis may be reversible. Zhang et al. [22] reported similar results, in that several hours of hypoxia caused obvious changes in expression of proteins related to tumor metastasis that gradually returned to baseline levels as the normoxic environment was reestablished. However, the different effects of transiently raising HIF-1 and VEGF on RCC proliferation and metastasis should not be ignored. HIF-1 $\alpha$ was reported to induce apoptosis of solid cancer cells by the p53 pathway [23, 24], whereas VEGF-D expression was correlated with the depth of tumor invasion and lymph node metastasis in human colorectal carcinoma [25]. The different effects of different pressures and concentrations for $\mathrm{CO}_{2}$ insufflation on RCC proliferation and metastasis should be noted. Bourdel et al. [26] reported that postoperative peritoneal dissemination of ovarian cancer cells is not promoted by a $\mathrm{CO}_{2}$ pneumoperitoneum with $2 \mathrm{~mm} \mathrm{Hg}$ intraperitoneal pressure in a mouse model when assessed on postoperative day 14 . Tan reported that the short-term effect of $\mathrm{CO}_{2}$ on transitional-cell carcinoma growth and apoptosis is complex, and high $\mathrm{CO}_{2}$ concentrations (> 5\%) and high insufflation pressures $(\geq 15 \mathrm{~mm} \mathrm{Hg}$ ) are most effective in reducing tumor-cell adhesion and growth [27].

In the present study, we mimicked $\mathrm{CO}_{2}$ pneumoperitoneum environments for treating RCC using an orthotropic xenograft model. At the cellular and tissue levels, $\mathrm{CO}_{2}$ pneumoperitoneum had no effect on RCC proliferation. At the protein level, $\mathrm{CO}_{2}$ pneumoperitoneum had a reversible effect on proteins related to renal cancer cell proliferation and metastasis.

Our study had two distinct limitations. We did not observe the growth rate of tumors in live mice which underwent creation of $\mathrm{CO}_{2}$ pneumoperitoneum vs those that did not. It was reported that renal cancer growth could be enhanced by pneumoperitoneum vs gassless procedure in rats [28].
Therefore, the growth rate of tumors in animal models which underwent creation of $\mathrm{CO}_{2}$ pneumoperitoneum needs to be further confirmed. The mouse model of pneumoperitoneum peritoneal inoculation of human line RCC is one of the possible ways of assessing safety of surgical treatment in humans, but it is not a typical human appearance of RCC, which is rather retroperitoneal and intrarenal. We did not design our study to investigate all these issues, so the relevant study needs to be conducted in future research.

In conclusion, in a human RCC orthotropic xenograft mouse model, $\mathrm{CO}_{2}$ pneumoperitoneum has no effect on the Ki67 cell proliferation marker. However, $\mathrm{CO}_{2}$ pneumoperitoneum rapidly induces HIF- $1 \alpha$ and VEGF expression, but these changes can be reversed upon resolution of hypoxia. Our results suggest that $\mathrm{CO}_{2}$ pneumoperitoneum laparoscopy may be a safe method for treating RCC.

\section{Acknowledgments}

Yuan-Zhuo Chen and Yun-Fei Xu - contributed equally to this work.

\section{References}

1. Peng $\mathrm{Y}$, Zheng $\mathrm{M}$, Ye $\mathrm{Q}$, et al. Heated and humidified $\mathrm{CO}_{2}$ prevents hypothermia, peritoneal injury, and intra-abdominal adhesions during prolonged laparoscopic insufflations. J Surg Res 2009; 151: 40-7.

2. Ost MC, Patel KP, Rastinehad AR, et al. Pneumoperitoneum with carbon dioxide inhibits macrophage tumor necrosis factor-alpha secretion: source of transitional-cell carcinoma port-site metastasis, with prophylactic irrigation strategies to decrease laparoscopic oncologic risks. J Endourol 2008; 22: 105-12.

3. Krause P, Bobisch NS, Thelen P, et al. The plasminogen activator inhibitor system in colon cancer cell lines is influenced by the CO2 pneumoperitoneum. Int J Colorectal Dis 2011; 26: 37-43.

4. Nakada H, Ishida H, Hashimoto D, et al. Influence of different pneumoperitoneal pressures on tumor cell distribution in rats. Surg Endosc 2005; 19: 563-8.

5. Leng J, Lang J, Jiang Y, et al. Impact of different pressures and exposure times of a simulated carbon dioxide pneumoperitoneum environment on proliferation and apoptosis of human ovarian cancer cell lines. Surg Endosc 2006; 20: 1556-9.

6. Azuar AS, Matsuzaki S, Darcha C, et al. Impact of surgical peritoneal environment on postoperative tumor growth and dissemination in a preimplanted tumor model. Surg Endosc 2009; 23: 1733-9.

7. Ma JJ, Feng $\mathrm{B}$, Zhang $\mathrm{Y}$, et al. Higher $\mathrm{CO}_{2}$-insufflation pressure inhibits the expression of adhesion molecules and the invasion potential of colon cancer cells. World J Gastroenterol 2009; 15: 2714-22.

8. Luo HX, Yu PW, Hao YX, et al. Effects of $\mathrm{CO}(2)$ pneumoperitoneum on peritoneal macrophage function and peritoneal metastasis in mice with gastric cancer. Eur Surg Res 2012; 48: 40-7.

9. Matsuzaki S, Bourdel N, Darcha C, et al. Molecular mechanisms underlying postoperative peritoneal tumor dissemination may differ between a laparotomy and car- 
bon dioxide pneumoperitoneum: a syngeneic mouse model with controlled respiratory support. Surg Endosc 2009; 23: 705-14.

10. Izumi K, Ishikawa K, Tojigamori M, et al. Liver metastasis and ICAM-1 mRNA expression in the liver after carbon dioxide pneumoperitoneum in a murine model. Surg Endosc 2005; 19: 1049-54.

11. Tomita H, Marcello PW, Milsom JW, et al. $\mathrm{CO}_{2}$ pneumoperitoneum does not enhance tumor growth and metastasis: study of a rat cecal wall inoculation model. Dis Colon Rectum 2001; 44: 1297-301.

12. Zwierzchowski TJ, Stasikowska-Kanicka O, Janus J, et al. Evidence for apoptosis, MMP-1, MMP-3 and TIMP-2 expression and their effect on the mechanical and biochemical properties of fresh viable knee medial meniscal allografts and autografts in the rabbit. Arch Med Sci 2012; 8: 724-32.

13. Ridgway PF, Smith A, Ziprin P, et al. Pneumoperitoneum augmented tumor invasiveness is abolished by matrix metalloproteinase blockade. Surg Endosc 2002; 16: 533-6.

14. Semenza GL. Regulation of oxygen homeostasis by hypoxia-inducible factor 1. Physiology (Bethesda) 2009; 24: 97-106.

15. Ke Q, Costa M. Hypoxia-inducible factor-1 (HIF-1). Mo Pharmacol 2006; 70: 1469-80.

16. Hu CJ, Wang LY, Chodosh LA, et al. Differential roles of hypoxia-inducible factor 1alpha (HIF-1alpha) and HIF-2alpha in hypoxic gene regulation. Mol Cell Biol 2003; 23: 9361-74.

17. Klatte T, Seligson DB, Riggs SB, et al. Hypoxia-inducible factor 1 alpha in clear cell renal cell carcinoma. Clin Cancer Res 2007; 13: 7388-93.

18. Li J, Diao H, Zhao D, et al. Reduced tumourigenicity of EG7 after RANTES gene transfer and the underlying mechanism. Arch Med Sci 2010; 6: 829-36.

19. Jalava P, Kuopio T, Juntti-Patinen L, et al. Ki67 immunohistochemistry: a valuable marker in prognostication but with a risk of misclassification: proliferation subgroups formed based on Ki67 immunoreactivity and standardized mitotic index. Histopathology 2006; 48: 674-82.

20. Wildbrett P, Oh A, Naundorf D, et al. Impact of laparoscopic gases on peritoneal microenvironment and essential parameters of cell function. Surg Endosc 2003 17: 78-82.

21. Cai KL, Wang GB, Xiong LJ. Effects of carbon dioxide and nitrogen on adhesive growth and expressions of E-cadherin and VEGF of human colon cancer cell CCL-228. World J Gastroenterol 2003; 9: 1594-7.

22. Zhang X, Guo X, Zhang A, et al. Influence of carbon dioxide pneumoperitoneum environment on adhesion and metastasis of a human ovarian cancer cell line. Surg Endosc 2009; 23: 108-12.

23. Rohwer N, Dame C, Haugstetter A, et al. Hypoxia-inducible factor 1 alpha determines gastric cancer chemosensitivity via modulation of p53 and NF-kappaB. PLoS One. 2010; 5: e12038.

24. Erdem H, Oktay M, Yildirim U, et al. Expression of AEG-1 and p53 and their clinicopathological significance in malignant lesions of renal cell carcinomas: a microarray study. Pol J Pathol 2013; 64: 28-32.

25. Nogawa S, Kitadai Y, Tanaka S, et al. Expression of VEGF-C and VEGF-D at the invasive edge correlates with lymph node metastasis and prognosis of patients with colorectal carcinoma. Cancer Sci 2004; 95: 32-9.
26. Bourdel N, Matsuzaki S, Bazin JE, et al. Postoperative peritoneal dissemination of ovarian cancer cells is not promoted by carbon-dioxide pneumoperitoneum at low intraperitoneal pressure in a syngenic mouse laparoscopic model with controlled respiratory support: a pilot study. J Minim Invasive Gynecol 2008; 15: 321-6.

27. Tan BJ. Is carbon dioxide insufflation safe for laparoscopic surgery? A model to assess the effects of carbon dioxide on transitional-cell carcinoma growth, apoptosis, and necrosis. J Endourol 2006; 20: 965-9.

28. Bouvy ND, Giuffrida MC, Tseng LN, et al. Effects of carbon dioxide pneumoperitoneum, air pneumoperitoneum, and gasless laparoscopy on body weight and tumor growth. Arch Surg 1998; 133: 652-6. 